\title{
Relationships between Safety Perception, Knowledge, and Compliance among Hospital Employees
}

\author{
Su Kyoung Chung1)
}

\begin{abstract}
This descriptive correlation study aimed to examine the relationship of safety knowledge, safety perception and safety compliance among hospital organization employees. The subjects were 223 self-reported questionnaires from nurses, interns and residents who worked between July and September of 2009 at a university hospital in Seoul. The data were analyzed using Pearson correlation coefficient and stepwise multiple regression. The relationship between safety knowledge, perception and compliance among hospital organization employees revealed to be significantly correlated each other $(p<.001)$. The most important factor to effect on safety compliance was safety knowledge $(\beta=.431, p<.001)$ and explained about $39.9 \%$ of the variance. If safety perception $(\beta=.382, p<.001)$ were added that safety compliance was explained about $50.2 \%$ of the variance. These findings suggested that comprehensive interventions for enhancing the safety compliance of hospital organization employees should be focused on developing the safety knowledge and the safety perception of hospital organization employees.
\end{abstract}

Keywords : Safety Perception, Safety Knowledge, Safety Compliance, Hospital Employees

\section{Introduction}

In recent years, interest in safety culture in hospitals has been growing, and hospitals have made various efforts to prevent safety accidents. Prevention is a priority because safety accidents can lead to financial loss, poor quality of care, or even death. Mortality and disability caused by medical accidents have only recently emerged as a topic of public discussion in South Korea[1] and the implementation of service rating systems for medical institutions has been a focus of the burgeoning interest in strategies for preventing safety accidents[2]. As a result of such heated interest, multiple hospitals have strengthened their efforts to increase their employees' safety compliance. Safety compliance refers to major safety activities that individuals must engage in to maintain safety on the job, including wearing safety protective devices and adhering to safety guidelines and policies[3].

Received(October 15, 2018), Review Result(1st: October 31, 2018, 2nd: November 30, 2018), Accepted(December 10, 2018)

1) (Associate Professor) 34606 Department of Nursing, Dongdaejeon-Ro 171, Woosong University, Dong-gu, Daejeon, Korea

email: csk6677@hanmail.net 
Industrial workers' safety knowledge was the first safety climate topic that researchers studied[4]. Managerial efforts have been made to improve employees' safety knowledge by providing safety training and education programs with the ultimate goal of increasing safety procedure compliance. Raising the level of knowledge of safety contributes to reducing accidents[5], and estimating the level of safety knowledge can lead to a $35 \%$ prediction of safety performance[6]. However, even if employees are willing and able to comply with safety regulations and guidelines, consistent safety performance and compliance would still be impossible if they lacked the relevant knowledge about how to do so. Studies have shown that rate at which accidents get reported in industrial workplaces is consistently lower when employees believe that their organizations prioritize safety. Employees' perception of safety has been identified as an important factor that affects workers' safety compliance[7][8] as it has been shown that increasing their perception of safety results in greater compliance with safety policies and regulations[9].

The concept of safety compliance has been actively promoted in industrial workplaces since the 1990s in foreign countries and has been actively introduced in hospital environments since the 2000s. Most safety-related studies involving Korean hospitals were mainly investigative studies that examined safety-related perceptions and attitudes[1][2] and studies on safety compliance mainly investigated the relationships among perceptions of safety climate, sense of safety control, and safety compliance[10] and the factors that affected compliance with standard precautions[11]. However, there have not been any studies conducted to identify which factors affect safety compliance.

The two most important concepts mentioned in relation to safety climate are perception and knowledge of safety. Shedding light on whether these factors affect safety compliance would give hospitals the foundation on which to establish efficient strategies for increasing their employees' safety compliance as a safety management measure. Therefore, this study investigated hospital employees' levels of safety perception, knowledge, and compliance and the relationships between these variable to provide baseline data for developing methods to improve safety compliance among South Korean hospital employees.

\section{Study Goal}

This study investigated the correlation between levels of safety perception, knowledge, and compliance among hospital employees to provide baseline data for establishing strategies to 
improve safety compliance among hospital employees. The specific objectives were to:

1) Investigate hospital employees' levels of safety perception, knowledge, and compliance;

2) Investigate the relationships between hospital employees' levels of safety perception, knowledge, and compliance; and

3) Identify the factors that affect hospital employees' safety compliance.

\section{Method}

\subsection{Study Design}

This study was a descriptive correlational study examining the hospital employees' levels of safety perception, knowledge, and compliance and the correlations between hospital employees.

\subsection{Subjects and Data Collection}

Data were collected from July to August 2009 from self-reporting questionnaires distributed to nurses, interns, and residents who worked at randomly chosen a university hospital in Seoul. Participation in this study was approved by the hospital's Institutional Review Board. Eight research assistants were trained and educated on data collection precautions and procedures. After obtaining informed consent from the participants, questionnaires were distributed and collected when completed. Out of 280 questionnaires given, 239 questionnaires were collected. The questionnaires were excluded under the suspicion that they represented insincere responses as indicated by marking the middle score for all items or extreme scores for all items. Thus, a total of 223 questionnaires were included in the analysis. According to the Cohen's G-power calculation Version 3.1.9.2, the number of samples required for each group to maintain a significance of .05 , an effect size of .15 (medium), and a power of .80 for a maximum of 10 variables for regression was 118. Thus, the sample size for each group was confirmed to be appropriate.

\subsection{Study Tools}

\subsubsection{Safety perception}


Safety perception was measured using eight items about safety-related priorities and patient orientation developed by Ramanujam, Abrahamson, and Anderson[12], including, "I prioritize patient safety when providing care (treat)," "I believe that I am responsible for improving patient safety," and "I believe that people who continually strive to ensure patient safety are respected." Each item was rated on a scale of 1 "Not at all" to 5 "Very true," resulting in a maximum possible score of 40 points. The reliability as measured by Cronbach's $a$ at the time of the development was .87 and was .62 in this study.

\subsubsection{Safety knowledge}

Safety knowledge was measured using three items about safety knowledge and policies during patient care (treat) developed by Probst and Brubaker[13], including, "I know exactly what to look for when the care (treat) method is unsafe and uncertain," "I know the safety regulations and guidelines needed when providing care (treat)," and four items about safety practices and guidelines developed by Neal, Griffin, and Hart[6], including "I know how to provide care (treat) that is safe for patients," and "I know how to use protective gear or safety devices." Each item was rated on a scale of 1 "Not at all" to 5 "Very true," resulting in a maximum possible score of 35 points. The Cronbach's a was 0.61 for the scale developed by Probst and Brubaker[13] and .90 for the scale developed by Neal, Griffin, and Hart[6] at the time of development. The Cronbach's a of the tool in this study was .86 .

\subsubsection{Safety compliance}

Safety compliance was measured using 11 items developed by Hayes, Perander, Smecko, and Trask[14], including, "I tend to ignore safety guidelines to complete my nursing tasks quickly," "I adhere to all safety guidelines regardless of the situation I am in," and "I encourage my colleagues to follow safety guidelines when providing care (treat)." Each item was rated on a scale of 1 "Not at all" to 5 "Very true," resulting in a maximum possible score of 55 points. The Cronbach's a of the tool at the time of development was .85 and was .79 in this study.

\subsubsection{Data Analysis}

All collected data were analyzed using SPSS Win 15.0 software. Descriptive statistics were generated for participants' demographic information and the study variables. Correlations between the variables were tested with Pearson's correlation coefficient and factors that were hypothesized to affect safety compliance were analyzed using stepwise multiple regression. 


\section{Results}

\subsection{Participant Characteristics}

The mean age of the participants was 29.1 years with a range of 22 - 49 years, and 75 male (34\%) and 148 female (66\%) were included. A total of 223 participants, composed of 109 nurses (49\%) and 114 physicians, were enrolled in this study. Physicians included $30(13 \%)$ interns and $84(38 \%)$ residents. The participants had an average clinical experience of 4.5 years [Table 1].

[Table 1] General Characteristics ( $\mathrm{N}=223)$

\begin{tabular}{|c|c|c|c|}
\hline Characteristics & Value & $\mathrm{n}(\%)$ & Mean \pm SD \\
\hline \multirow{3}{*}{ Age(yr) } & $22-30$ & 161(72) & \multirow{3}{*}{$29.16 \pm 4.29$} \\
\hline & $31-40$ & $57(26)$ & \\
\hline & $41-50$ & $5(2)$ & \\
\hline \multirow{2}{*}{ Gender } & male & $75(34)$ & \\
\hline & female & $148(66)$ & \\
\hline \multirow{3}{*}{ Job } & nurse & $109(49)$ & \\
\hline & intern & $30(13)$ & \\
\hline & resident & $84(38)$ & \\
\hline \multirow{4}{*}{$\begin{array}{c}\text { Resident } \\
\text { training } \\
\text { experience(year) }\end{array}$} & 1 & $33(38)$ & \\
\hline & 2 & $23(26)$ & \\
\hline & 3 & $23(26)$ & \\
\hline & 4 & $9(10)$ & \\
\hline \multirow{4}{*}{$\begin{array}{c}\text { Clinical } \\
\text { experience(year) }\end{array}$} & 5 or less & $165(74)$ & \multirow{4}{*}{$4.5 \pm 4.7$} \\
\hline & $6-10$ & $33(15)$ & \\
\hline & $11-15$ & 11(5) & \\
\hline & 15 or more & $14(6)$ & \\
\hline
\end{tabular}

\subsection{Levels of Safety Perception, Knowledge, and Compliance}

Descriptive statistics for this study are shown in [Table 2]. Safety perception scores had a range of 18-39 with a mean score of 28.02. The mean score for each safety perception item was 3.36. The highest score was for "I prioritize patient safety when providing nursing service" with a mean score of 3.82 points while the lowest score was for "I frequently take time to explore safe methods of providing patient care (treat)" with a mean score of 3.06 points. Safety knowledge scores had a range of 13 - 35 with a mean score of 23.69 points. The mean score for each safety knowledge item was 3.38. The highest score was for "I know how to provide care (treat) that is safe for patients" with a mean score of 3.82 points while the lowest score 
was for "I actively demand more safety training when necessary" with a mean score of 2.94 points. Safety compliance scores had a range of $22-51$ with a mean score of 37.65 points. The mean score for each safety compliance item was 3.18. The highest score was for "I maintain a clean environment when providing care (treat)" with a mean score of 3.74 points while the lowest score was for "I adhere to all safety guidelines regardless of the situation I am in" with a mean score of 3.11 points.

[Table 2] Mean Score of the Study Variables $(\mathrm{N}=223)$

\begin{tabular}{|l|c|c|c|c|c|}
\hline & Mean \pm S.D & Min & Max & Mean \pm S.D & Item mean \pm S.D \\
\hline Safety perception & $28.02 \pm 3.28$ & 18 & 39 & $28.02 \pm 3.28$ & $3.36 \pm .41$ \\
\hline Safety knowledge & $23.69 \pm 3.86$ & 13 & 35 & $23.69 \pm 3.86$ & $3.38 \pm .55$ \\
\hline Safety compliance & $37.65 \pm 5.33$ & 22 & 51 & $37.65 \pm 5.32$ & $3.18 \pm .32$ \\
\hline
\end{tabular}

\subsection{Correlations between Levels of Safety Perception, Knowledge, and Compliance}

The correlations between hospital employees' demographic information, levels of safety perception, levels of safety knowledge, and levels of safety compliance are shown in [Table 3]. Length of clinical career was statistically significantly positively correlated with safety perception $(\mathrm{r}=0.415, \mathrm{p}<.001)$, knowledge $(\mathrm{r}=.481, \mathrm{p}<.001)$, and compliance $(\mathrm{r}=.382, \mathrm{p}<.001)$. Safety perception was statistically significantly positively correlated with safety knowledge ( $\mathrm{r}=.531$, $\mathrm{p}<.001)$ and compliance $(\mathrm{r}=.610, \mathrm{p}<.001)$ and safety knowledge and compliance were statistically significantly positively correlated with each other $(\mathrm{r}=.634, \mathrm{p}<.001)$.

[Table 3] Relationship between the Study Variables ( $\mathrm{N}=223$ )

\begin{tabular}{|c|c|c|c|}
\hline & Clinical experience & Safety perception & Safety knowledge \\
\hline Clinical experience & 1 & & \\
\hline Safety perception & $.415(<.001)$ & 1 & 1 \\
\hline Safety knowledge & $.481(<.001)$ & $.531(<.001)$ & $.634(<.001)$ \\
\hline Safety compliance & $.382(<.001)$ & $.610(<.001)$ & \\
\hline
\end{tabular}

\subsection{Factors that Affected Hospital Employees' Safety Compliance}

Stepwise multiple regression was performed to identify the factors that affected hospital employees' safety compliance [Table 4]. Participants' clinical career data were converted to 
dummy variables for the purpose of conducting the analysis. Of the categories of demographic information, clinical career was excluded given the nature of the stepwise regression. The variable that most affected safety compliance was safety knowledge $(\beta=.431, p<.001)$, which accounted for $39.9 \%$ of the variance of safety compliance. Safety knowledge and perception together accounted for $50.2 \%$ of the variance $(\beta=.382, p<.001)$.

[Table 4] Factors Influencing of Safety Compliance ( $\mathrm{N}=223$ )

\begin{tabular}{|c|c|c|c|c|c|c|c|}
\hline & $\mathrm{B}$ & $\mathrm{S} . \mathrm{E}$ & $\beta$ & adjusted R2 & $\mathrm{t}$ & $\mathrm{p}$ & $\mathrm{F}(\mathrm{p})$ \\
\hline constant & 6.261 & 2.217 & & & 2.824 & .005 & $112.736(.000)$ \\
\hline Safety knowledge & .594 & .077 & .431 & .399 & 7.712 & .000 & \\
\hline Safety perception & .618 & .091 & .382 & .502 & 6.828 & .000 & \\
\hline
\end{tabular}

\section{Discussion}

This study investigated South Korean hospital employees' levels of safety perception, knowledge, and compliance, examined the degree to which they were correlated with each other, and identified the predictors of safety compliance.

In this study, the mean score for safety perception items was 3.36. The highest-rated item "I prioritize patient safety when providing nursing service" received a mean score of 3.82 points, which was relatively high. The lowest-rated item was "I frequently take time to explore safe patient care (treat) methods" which received a mean score of 3.06 points. Overall, the mean scores of all of the safety perception items were slightly above "neutral," highlighting the need for strategies to improve safety perception. Managerial competence is critical for improving safety perception, because managers directly impact the degree to which their employees pay attention to safety issues and increase their safety knowledge and perception[15]. Thus, strategies that improve managerial competence with regard to safety are also needed. The mean score for safety knowledge items was 3.38, which was lower than the 4.20 mean score reported by Neal, Griffin, and Hart[6] in their survey of the staff of a large Australian hospital and lower than the 3.96 and 4.02 mean scores reported by Probst and Brubaker[13] in their surveys of the employees of two food processing plants. Although the mean score for safety knowledge was slightly above "neutral" in this study, the fact that it was lower than scores reported in some studies indicates that strategies should be developed for how to educate hospital employees and improve their knowledge about safety policies, practices, and guidelines. The mean score for safety compliance items was 3.18, which was slightly above "neutral," but was lower than the average score of 4.22 reported by Hayes, Perander, Smecko, and Trask[14] in 
their survey of internal medicine residents. This result indicates that strategies should be developed for how to increase safety compliance, such as encouraging employees to maintain a clean environment, wear protective equipment, and adhere to safety guidelines in all situations.

There was a statistically significant positive correlation among levels of safety perception, knowledge, and compliance. Levels of safety compliance increased as levels of safety perception and knowledge increased. There was a positive correlation between levels of safety perception and knowledge, which was similar to the findings from the study conducted by Probst and Brubaker[13]. This study also found a positive correlation between safety perception and compliance, but Probst and Brubaker[13] did not. This inconsistency indicates that there is a need to conduct replication studies to shed light on the relationship between these two variables. A significant positive correlation was found between safety knowledge and compliance, which was similar to the results found in the studies by Neal, Griffin, and Hart[6], Probst[16], and Probst and Brubaker[13]. Thus, this study's findings found that safety knowledge is positively correlated with safety compliance.

In this study, safety knowledge was the strongest predictor of safety compliance, which was consistent with findings of the Probst and Brubaker[13] study. A South Korean study on industrial workplaces[5] found that safety knowledge reduced the number of different types of injuries experienced in the workplace. These findings indicate that strategies should be developed to increase hospital employees' safety knowledge. To this end, organizations that provide safety training should continuously update their programs to reflect actual risks and issues related to special tasks and develop and actively enforce training and education programs to improve employees' safety compliance. Furthermore, strategies and management systems for promoting the sharing of and knowledge about safety-related hospital policies and regulations are also needed.

In summary, there was a statistically significant relationship between hospital employees' safety perception, knowledge, and compliance. Organizational-level training programs, education programs, and strategies to increase managerial competence and improve environmental factors are needed to increase safety knowledge and perception in order to ultimately improve safety compliance.

There are several ways that future studies can expand upon this study's groundwork. Safety knowledge was found to increase hospital staff safety compliance. Thus, future studies should develop a relevant intervention program and assess its direct and indirect effects on safety compliance. In addition, future studies should also employ objective dependent variables in addition to self-reported safety compliance indicators. This study identified factors that affect 
safety compliance using a self-reported questionnaire, but qualitative studies should be performed as in-depth analyses of the variables that affect safety compliance.

\section{Conclusion}

This study had a descriptive correlational design to examine hospital employees' levels of safety perception, knowledge, and compliance and identify the relationships between them so as to provide baseline data for developing strategies to improve hospital employees' safety compliance.

The mean scores for hospital employees' levels of safety perception, knowledge, and compliance were 3.36, 3.38, and 3.18, respectively. Of employees' demographics, clinical career length had a statistically significant positive correlation with safety perception, knowledge, and compliance $(\mathrm{p}<.001)$. Furthermore, the correlations between hospital employees' safety perception, knowledge, and compliance were all statistically significantly positive $(\mathrm{p}<0.001)$. Safety knowledge had the greatest effect on safety compliance $(\beta=.431, p<.001)$, accounting for $39.9 \%$ of its variance. Safety knowledge and perception together $(\beta=.382, p<.001)$ explained $50.2 \%$ of the total variance in safety compliance.

Based on the finding that hospital employees' safety perception, knowledge, and compliance are statistically significantly positively correlated, organizational-level training, education programs, and strategies to increase managerial competence and improve environmental factors are needed to increase safety knowledge and safety perception with the ultimate goal of improving safety compliance.

\section{Acknowledgement}

This paper is the second analysis of the author's doctoral dissertation data with research support from Woosong University.

\section{References}

[1] M. A. Kang, J. E. Kim, G. A. Ahn, Y. Kim, and S. H. Kim, Physicians' perception of and attitudes toward patient safety culture and medical error reporting, Korean Journal of Health Polish \& Administration, (2005), Vol.5, No.4, pp.110-135. 
[2] J. E. Kim, M. A. Kang, G. A. Ahan, and Y. H. Sung, A Survey of Nurses' Perception of Patient Safety Related to Hospital Culture and Reports of Medical Errors, Clinical Nursing Research, (2007), Vol.13, No.3, pp.169-179.

[3] J. Mullen, Investing factors that influence individual safety behaviour at work, Journal of Safety Research, (2004), Vol.35, pp.275-285.

[4] D. Zohar, Safety climate in industrial organizations: theoretical and applied implications, Journal of Applied Psychology, (1980), Vol.65, pp.96-102.

[5] K. Y. Ahn, Moderating effect of age and relationship of safety climate and safety outcomes, Journal of Korean Society of Safety, (2005), Vol.20, No.4, pp.122-129.

[6] M. A. Griffin, A. Neal, and P. M. Hart, The impact of organizational climate on safety climate and individual behavior, Safety of Science, (2000), Vol.34, pp.99-109.

[7] M. D. Cooper, R. A. Phillips, Exploratory analysis of the safety climate and safety behaviour, Journal of Safety Research, (2003), Vol.35, pp.497-512.

[8] S. Silva, M. L. Lima, and C. Baptista, OSCI: an organisational and safety climate inventory, Safety Science, (2004), Vol.42, pp.205-220.

[9] C. Bailey, Managerial factors related to safety program effectiveness: an update on the Minnesota Perception Survey, Professional Safety, (1997), Vol.8, pp.33-35.

[10] Y-L. Kim, M-I. Lee, and E-Y. Chin, Convergence Effects of Nurse's Perception of Patient Safety Culture and Safety Control on Safety Performance in General Hospital, Journal of Digital Convergence, (2016), Vol.14, No.7, pp.201-211.

[11] M-S. Park, Exposure to Blood and Body Fluids and Influencing Factors on Compliance with Standard Precautions among Nurses in General Hospital, Journal of Korea Academia-Industrial Cooperation Society, (2016), Vol.17, No.11, pp.563-572.

[12] R. Ramanujam, K. Abrahamson, and J. A. Anderson, Influences on Nurse Perception of Hospital Unit Safety Climate: an HLM Approach, RCHE Publications, (2007), http://docs.lib.purdue.edu/rche_rp/34/, Oct 16 (2018).

[13] T. M. Probst, T. L. Brubaker, The Effects of Job Insecurity on Employee Safety Outcomes: Cross-Sectional and Longitudinal Explorations, Journal of Occupational Health Psychology, (2001), Vol.6, No.2. pp.139-159.

[14] B. E. Hayes, J. Perander, T. Smecko, and J. Trask, Measuring perceptions of workplace safety: Development and validation of the work safety scale, Journal of Safety Research, (1998), Vol.29, No.3, pp.145-161.

[15] J. Barling, C. Loughlin, and E. K. Kelloway, Development and Test of a Model Linking Safety-Specific Transformational Leadership and Occupational Safety, Journal of Applied Psychology, (2002), Vol.87, No.3, pp.488-496.

[16] T. M. Probst, Safety and insecurity: exploring the moderating effect of organizational safety climate, Journal of occupational health psychology, (2004), Vol.9, No.1, pp.3-10. 